\title{
Female disadvantage in the Egyptian labor market: A youth perspective [Arabic]
}

Maia Sieverding

Population Council

Follow this and additional works at: https://knowledgecommons.popcouncil.org/departments_sbsr-pgy

Part of the Demography, Population, and Ecology Commons, Family, Life Course, and Society Commons, International Public Health Commons, Labor Economics Commons, Women's Health Commons, and the Work, Economy and Organizations Commons How does access to this work benefit you? Let us know!

\section{Recommended Citation}

Sieverding, Maia. 2012. "Female disadvantage in the Egyptian labor market: A youth perspective [Arabic]," Survey of Young People in Egypt Policy Brief no. 4. Cairo: Population Council. 


$$
\text { مسبح النشء والشباب في مصدر }
$$

ملخـص السـياسـات رقهم ع
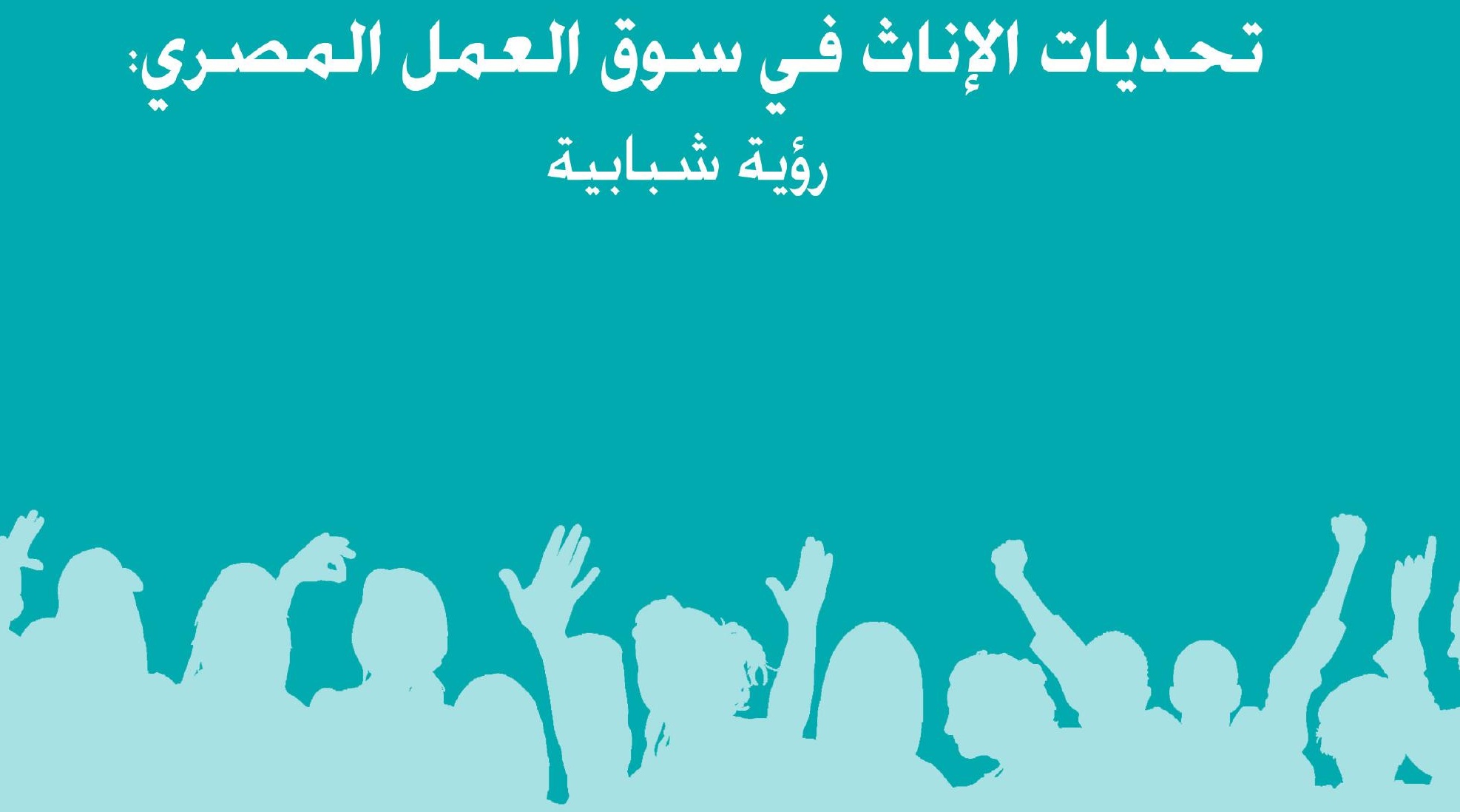

مايا سيفردينج

(1) Population Council

Research that makes a difference
FORDFOUNDATION

Working with Visionaries on the

Frontlines of Social Change Worldwide 


\section{استـتمرار تدني المشـاركتة في قوة العـمل بين \\ الفتيات}

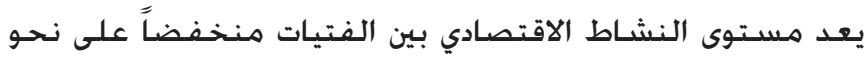

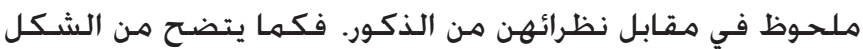

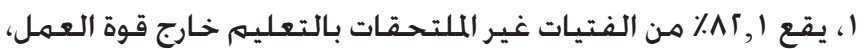

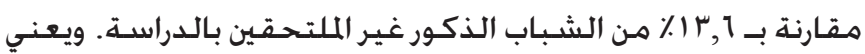

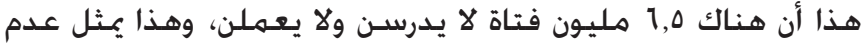

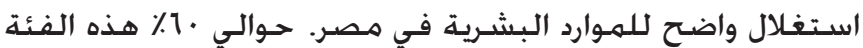

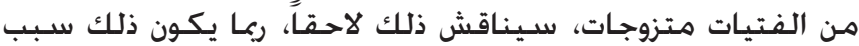

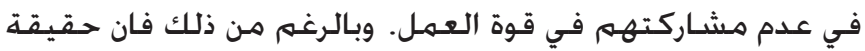

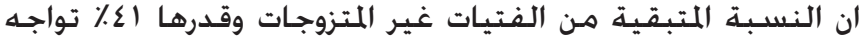

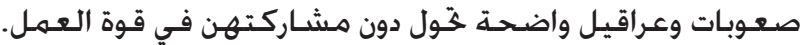

والتفاوت الواضح في نسبة المشاركة في قوة العمل بين الشباب

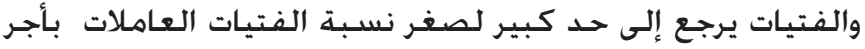

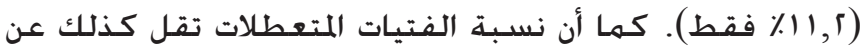

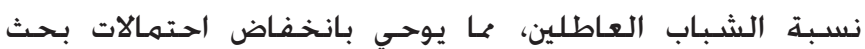

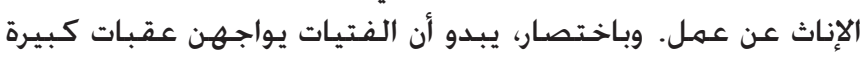

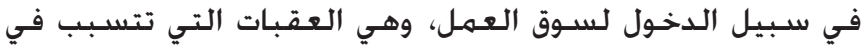
انخفاض كبير في قوة العمل المنتجـة في مصر.

الشكل ا: نسبة المشاركة في قوة العمل للأفراد غير الملتحقين

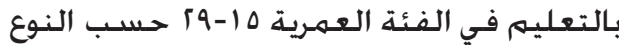

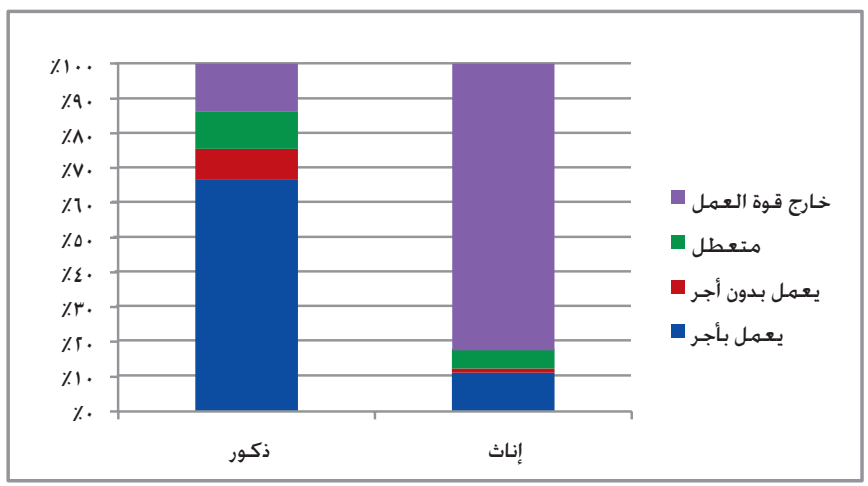

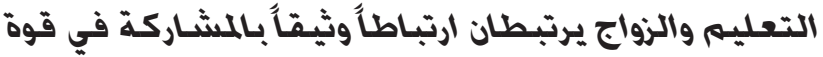

العمل التعل

يعد التعليم أحد المحددات الأسـاسية لمشاركة الإناث في قوة العمل

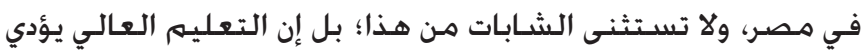

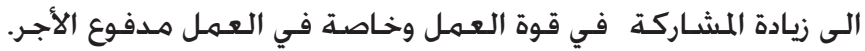

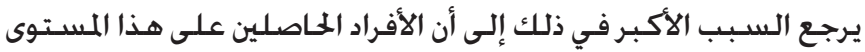

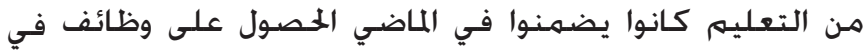

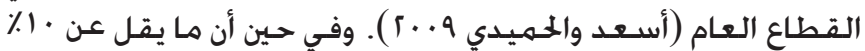

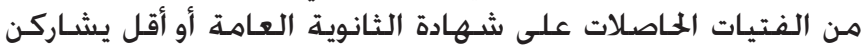

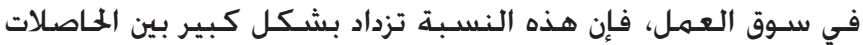

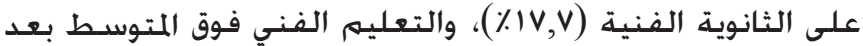

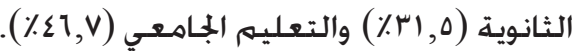

التقرير العالمي للفجوة بين الجنسين لعامٍ

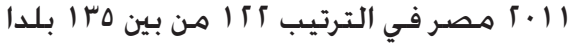

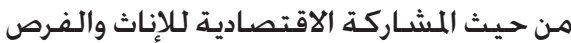

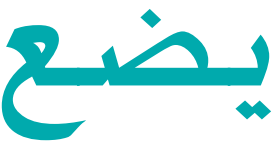
المتاحة لها. ويرجع هذا الترتيب إلى التحديات التي تواجهـها الإناث

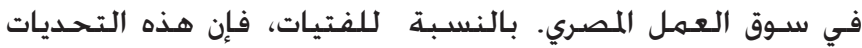

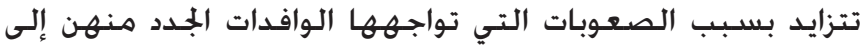

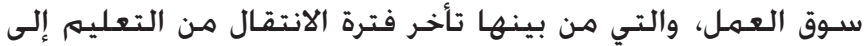

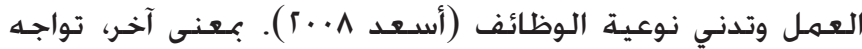

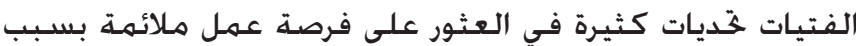
كونها انثى بل وصغيرة السـن.

وهذه التحديات المزدوجـة التي تعـانيها الفتيات في سـوق العمل بمصر

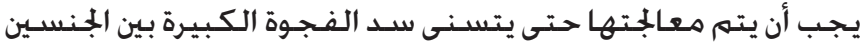

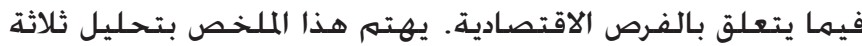

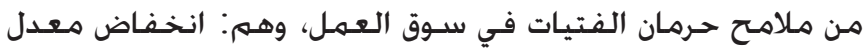

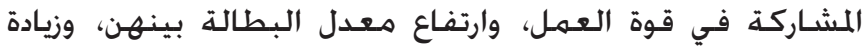

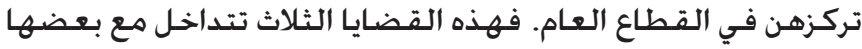

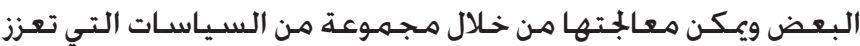

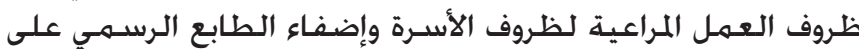

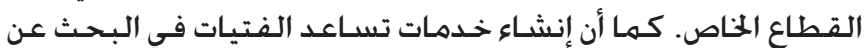

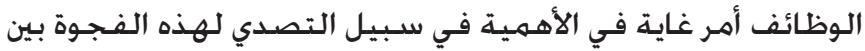
الجنسين فيما يتعلق بالبطالة.

\section{الرسـائل الأسـاسية}

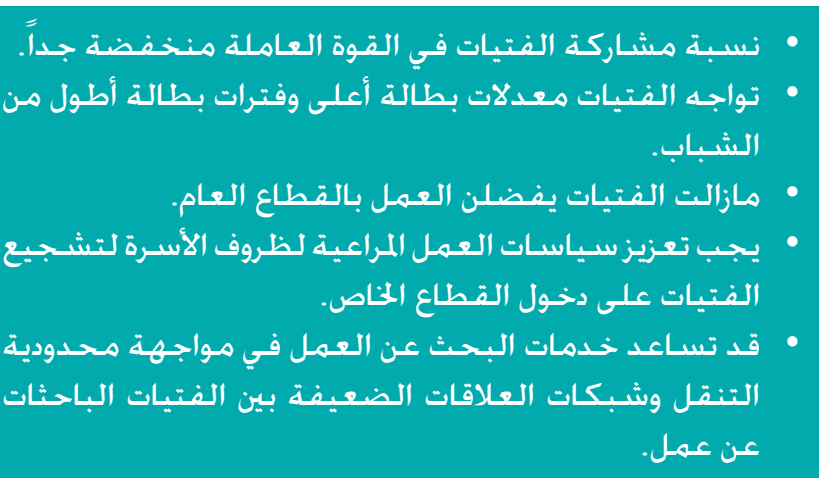

\section{البيانات والمنهجية}

ترتكز النتائج والتوصيات المقدمة في هذا الملخص على مخليل بيانات

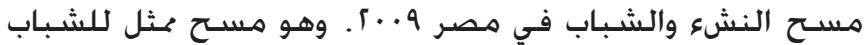

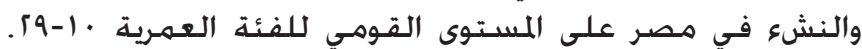

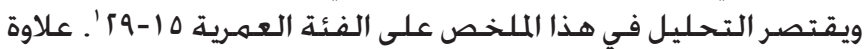

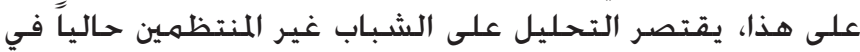

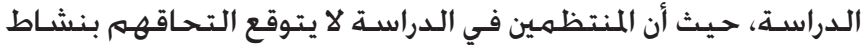

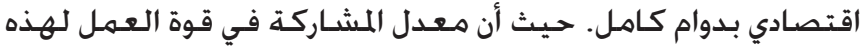

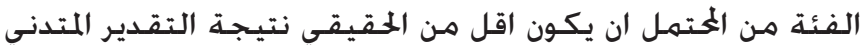
لاعدادهم.

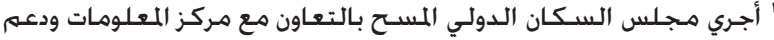

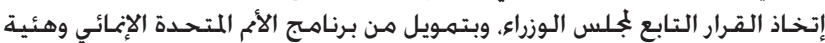

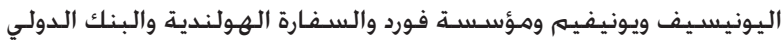

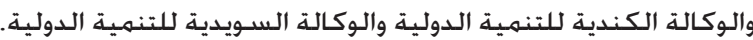




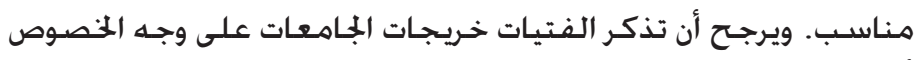

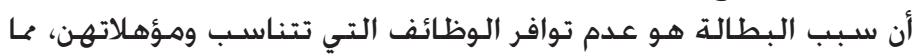

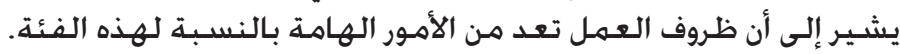

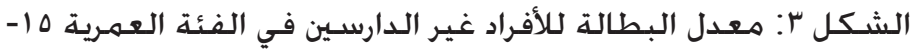

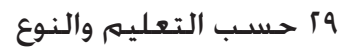

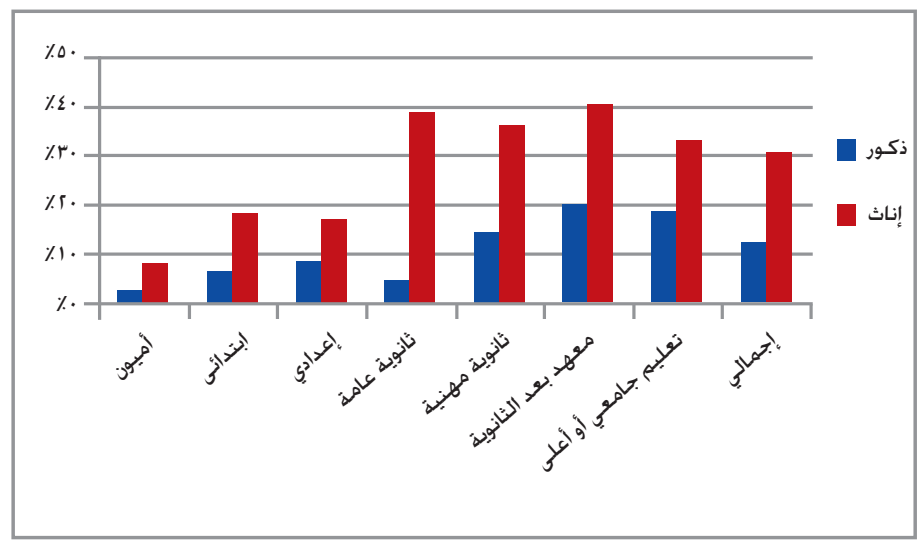

\section{بطالة الفتيـات تسـتمـر أطول مـن بـطالتة الشـبـاب}

إضـافـة إلى معاناة الفتيات من ارتفـاع معدلات البطالة، فهـن يواجهـن

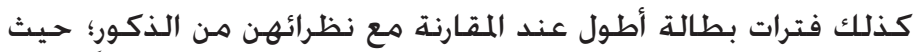

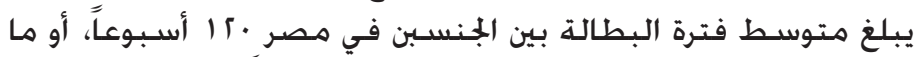

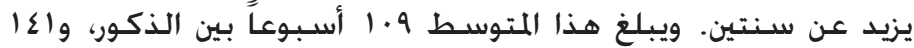

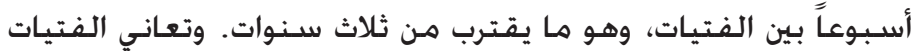

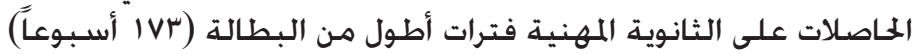

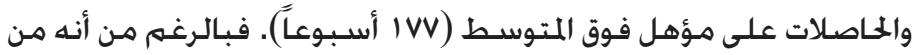

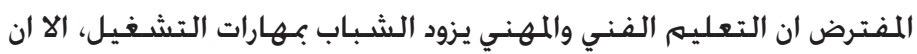

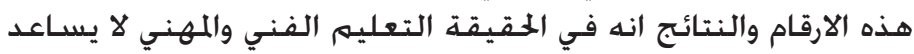

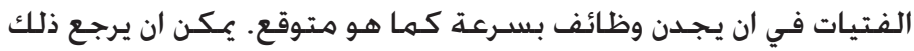

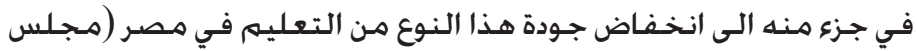

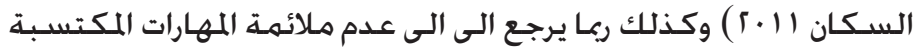

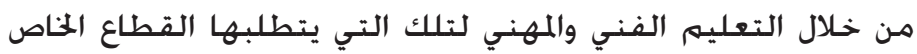

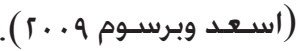

\section{يبلغ متوسـ طول فترة البطالة بين الفتيات V, أسنـة.}

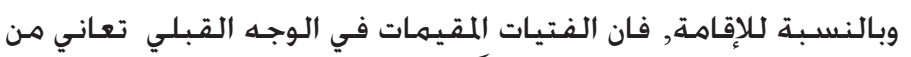

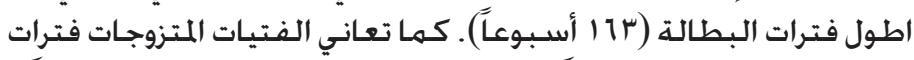

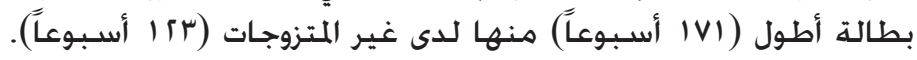

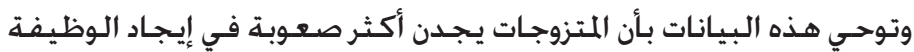
التي يرغبونها.

\section{الفتيات يعانين من قلة شبكة العلاقات ومحدودية التنقل في

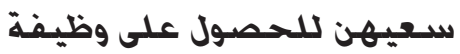

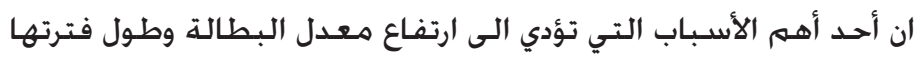

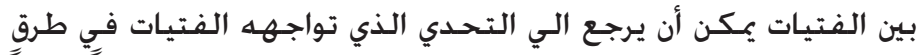

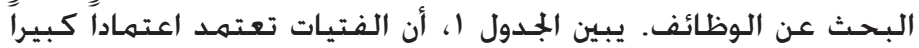

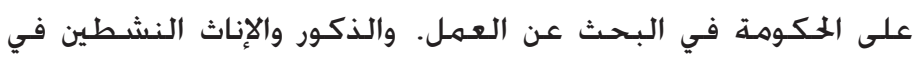

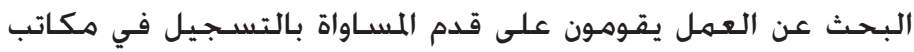

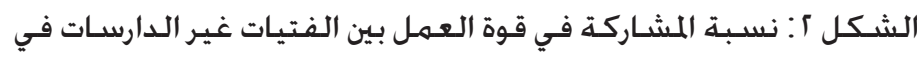

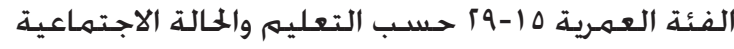

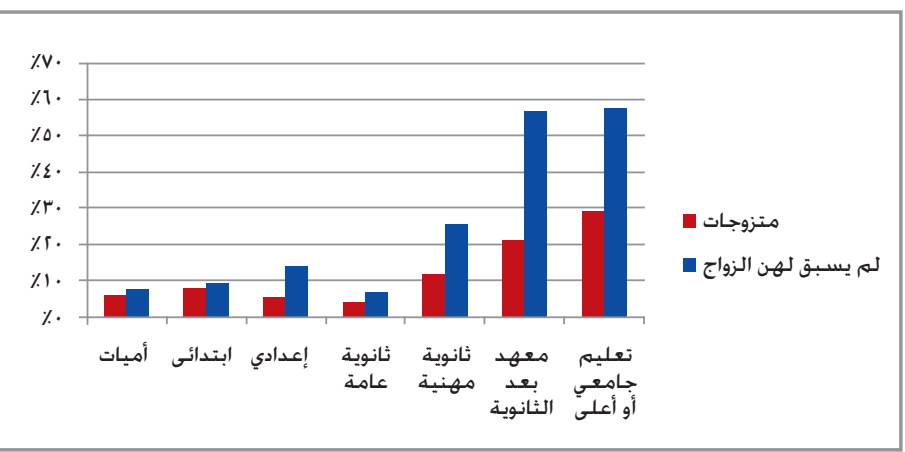

وإضافة إلى التعليه، يرتبط الزواج ارتباطاً وثيقاً بمشاركة الفتيات في تئي

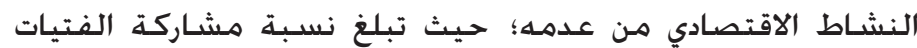

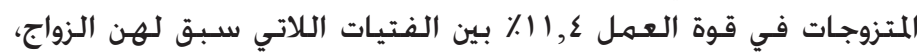

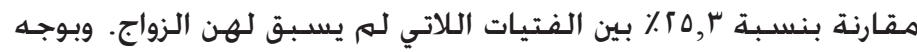

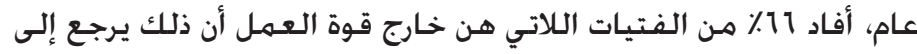

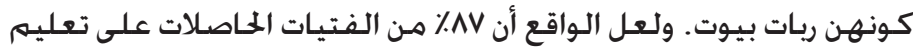

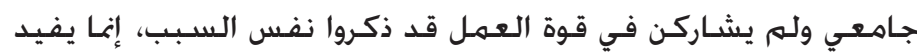

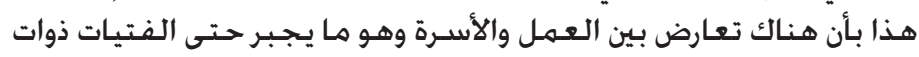

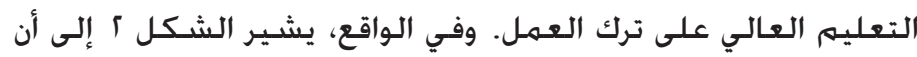

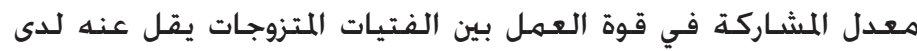

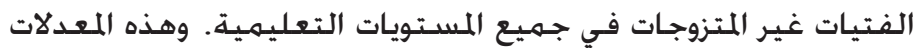

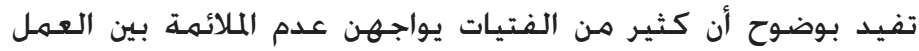
والأسـرة.

\section{تعاني الفتيـات معدلات بطالة أعلى من الشبـباب}

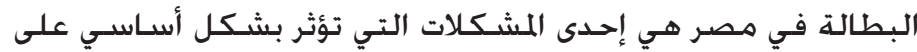

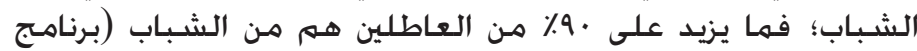

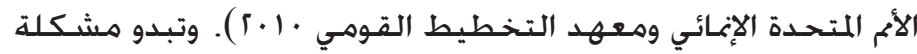

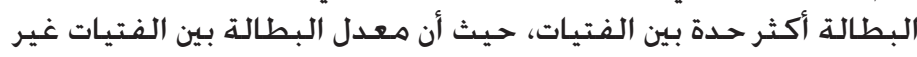

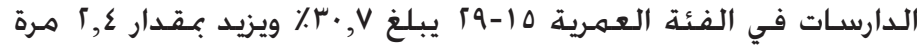

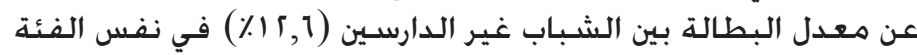

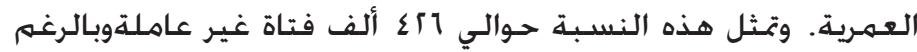

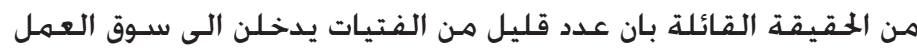

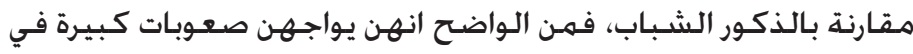

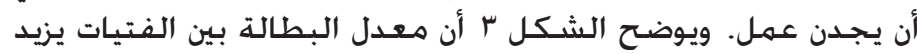

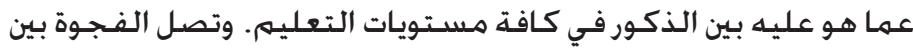

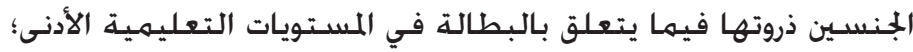

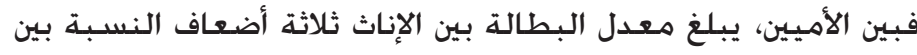

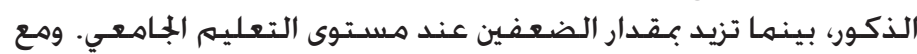

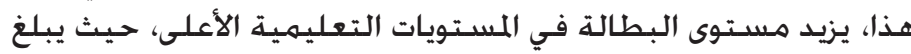
• ؛ بين خريجات معاهد ما بعد المرحلة الثانوية.

ويرجح أن معدلات البطالة تزداد بين الشباب الأكثر تعليماً بسبب الشبه

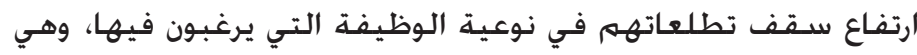

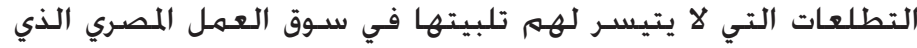

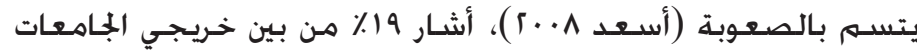

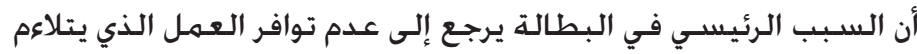

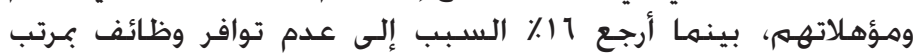


الشـكل ع- ا: الحالة العملية بين الذكور

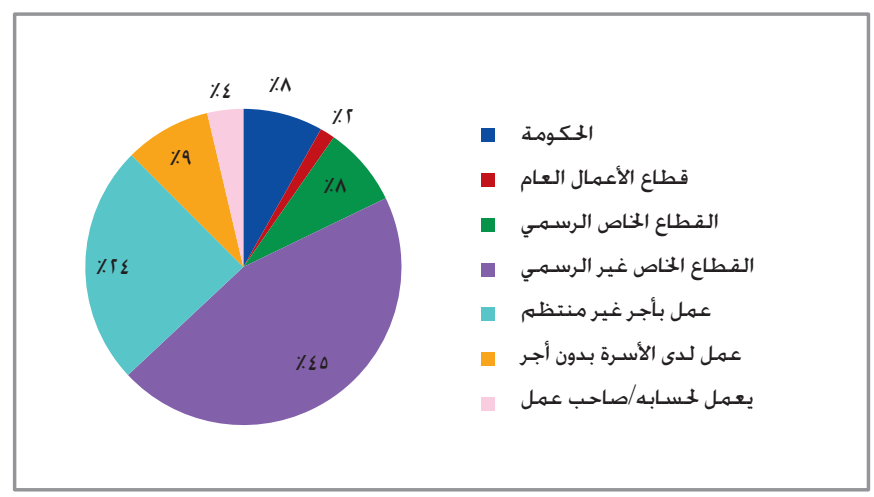

الشـكل ع-؟ ا: الحالة العـملية بين الفتيات

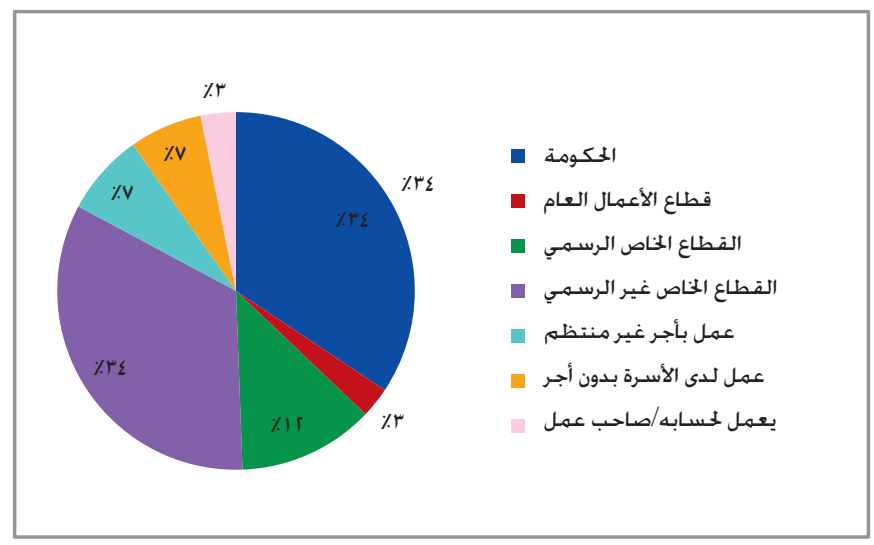

أقرت أغلب الفتيات بسـوء ظروف العمل في القطاع الحتاص

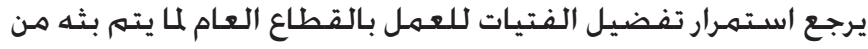

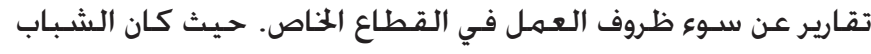

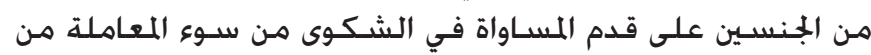

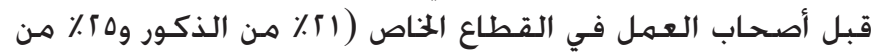

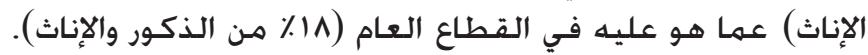

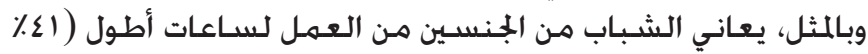

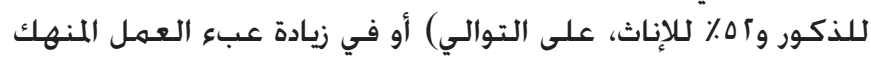

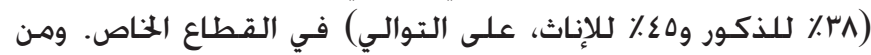

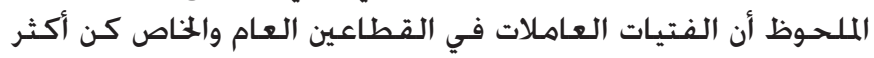

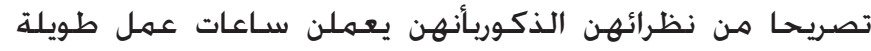

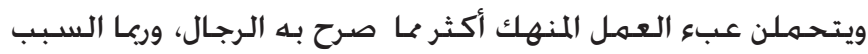
في ذلك يرجع الى اضطرارهم لتولي عبء العـمل المنزلي الإضـافي.

بالاضـافة الى ذلك، أفادت ؟1٪ من الفتيات العاملات بأنهـن تعرضوا

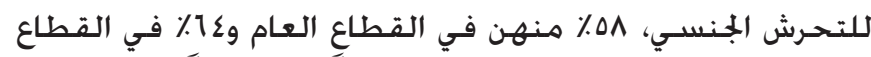

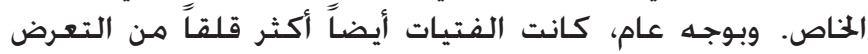

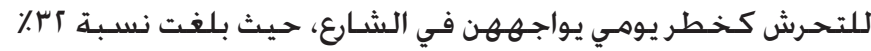

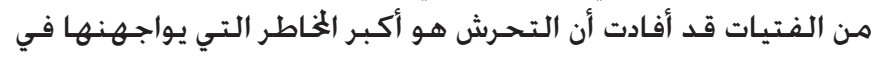

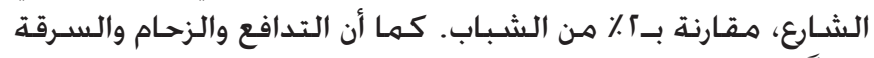

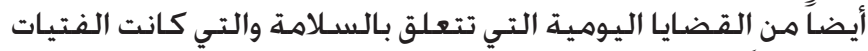

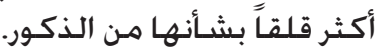

التوظيف الخاصة، ولكن الفتيات هن الأكثر تسجيلأ في المكاتب

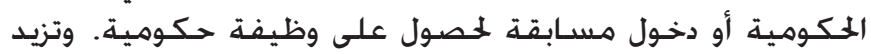

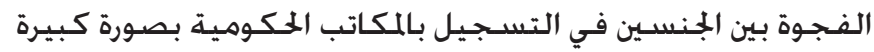

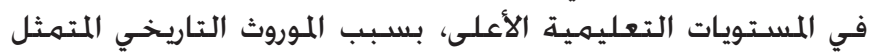

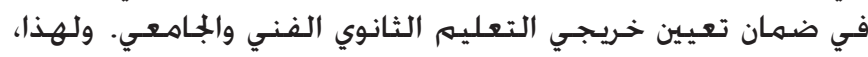

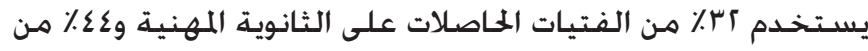

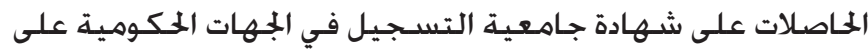

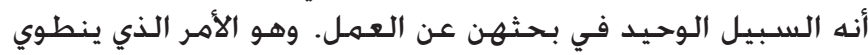

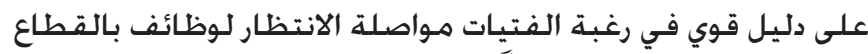

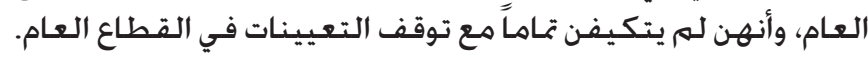

\begin{tabular}{|c|c|c|}
\hline \multicolumn{3}{|c|}{ 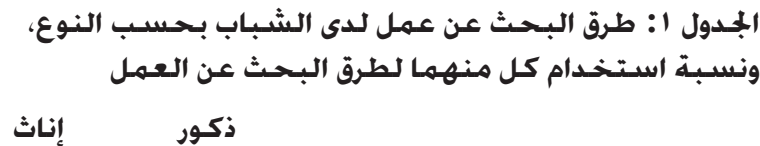 } \\
\hline $11, \cdot$ & $11, r$ & التســــل في القطـاع الخاص \\
\hline$r \Delta, \Delta$ & $1 \varepsilon, \varepsilon$ & التســيل في القطاع الحكـومي \\
\hline$\Delta \cdot, V$ & $1 \mathrm{H}, \mathrm{V}$ & دخـول مسـابقـة على وظيفـة حكـومية \\
\hline$\Sigma \Gamma, V$ & $r a, \Lambda$ & إرسـال طلب عمل \\
\hline 10, & $r \Sigma, r$ & الاســفسـار في مكان العـمل \\
\hline$\vee, \wedge$ & $\Gamma 1, \wedge$ & التعاقد مع صاحب عـمل \\
\hline$r 1,0$ & $1 \cdot, \varepsilon$ & طلأقب مسـاعدة مـن أحـــ الأصدقاء أو \\
\hline$r$ rev & $\Sigma V, \Sigma$ & الطــــــام الهـاتف الأرضـي في أي مـن \\
\hline$r \cdot, 1$ & $\varepsilon \Delta, 1$ & الطـــــــام الـهاتف العــــول في أي مـن \\
\hline
\end{tabular}

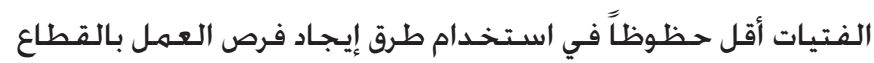

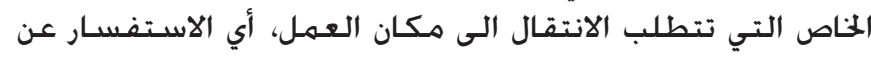

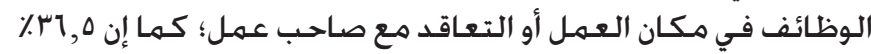

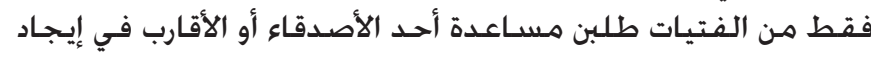

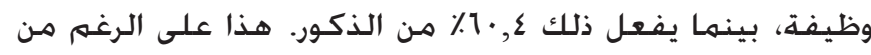

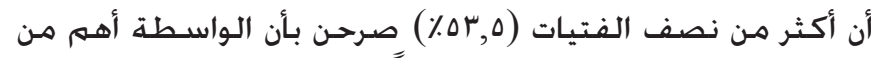

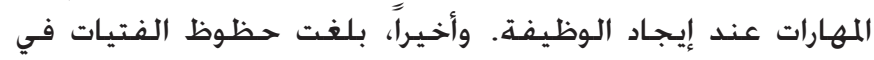

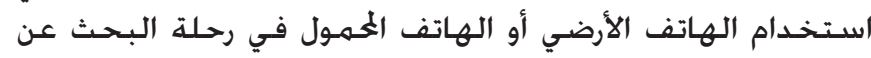

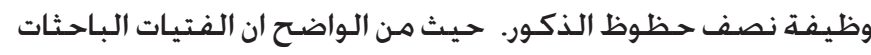

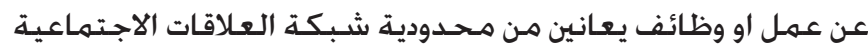

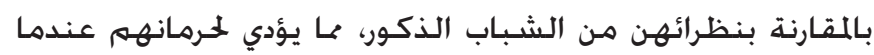

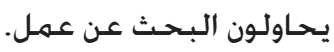

\section{يتركز عمل الفتيات في القطاع الحكـومي}

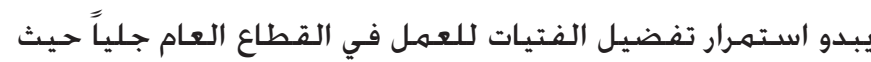

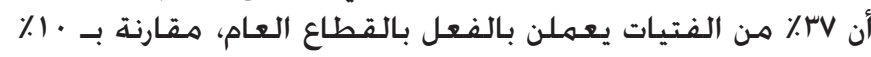

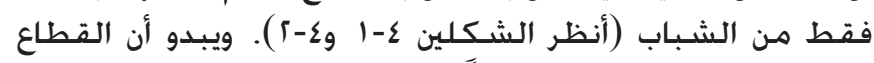

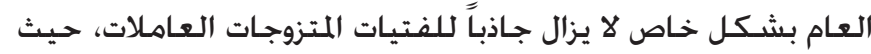

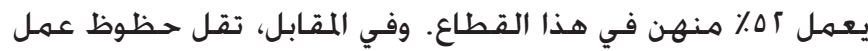

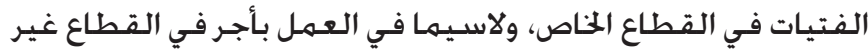

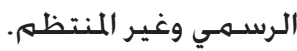


الخاص قـد يسـاعد في خـلق مناخ عمل أكثر تشـيعاً للفتيات.

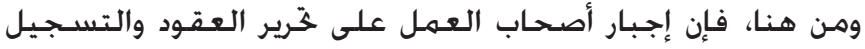

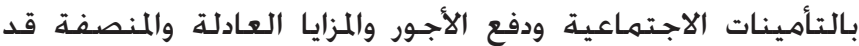

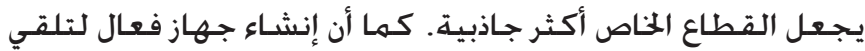

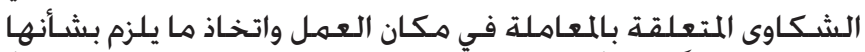

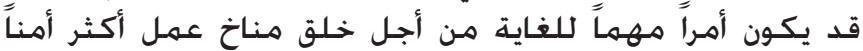

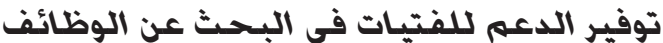

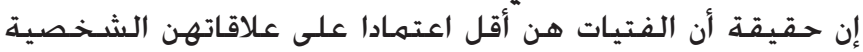

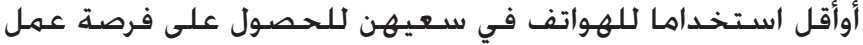

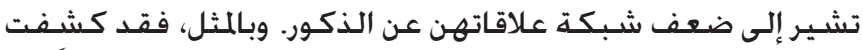

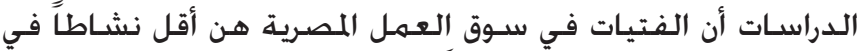

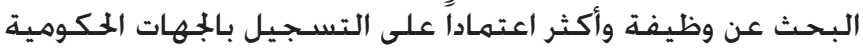

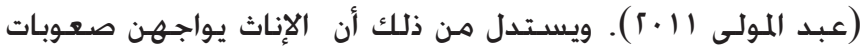

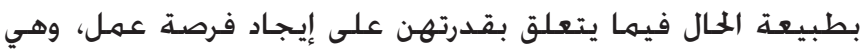

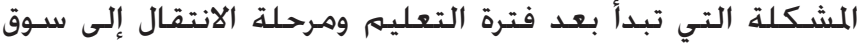

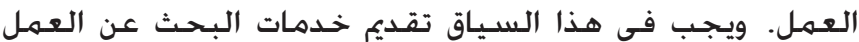

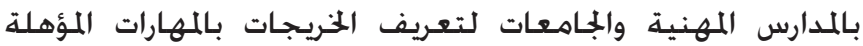

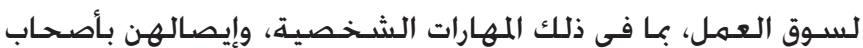

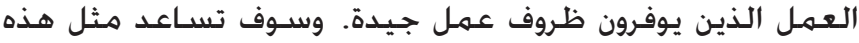

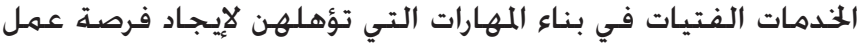

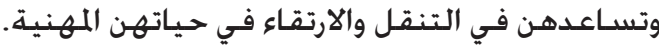

\section{المراجع}

Abdel Mowla, Somaya. 2011. Women's Job Search Behavior in the Egyptian Labor Market. Gender and Work in the MENA Region Working Paper Series: Poverty, Job Quality and Labor Market Dynamics, No. 21. Cairo: Population Council.

Assaad, Ragui. 2008. "Unemployment and Youth Insertion in the Labor Market in Egypt." In The Egyptian economy: Current challenges and future prospects. Ed. Hana Khayr al-Din, pp. 133-178. Cairo: American University in Cairo Press.

Assaad, Ragui and Ghada Barsoum. 2009. "Rising Expectations and Diminishing Opportunities for Egypt's Youth." In Generation in Waiting: The Unfulfilled Promise of Young People in the Middle East. Eds. Navtej Dhillon and Tarik Yousef, pp.67-94. Washington, D.C.: Brookings Institution.

Assaad, Ragui, and Fatma El Hamidi. 2009. "Women in the Egyptian Labor Market: An Analysis of Developments, 1988-2006." In The Egyptian Labor Market Revisited. Ed. Ragui Assaad, pp. 117-156. Cairo: American University in Cairo Press.

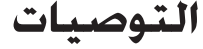

ترتبط الملامـح الثلاث للتحـدي الذي يواجهـ الفتيات في سـوق العـمل فئل

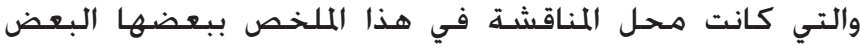

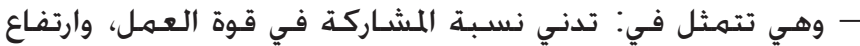

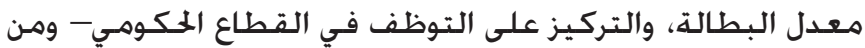

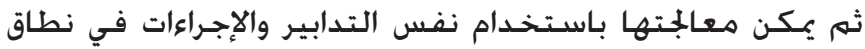

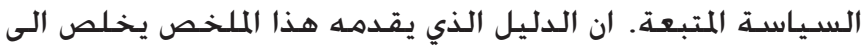

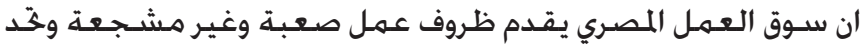

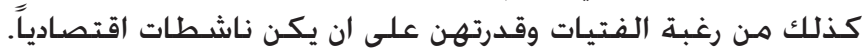

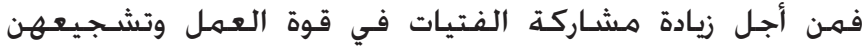

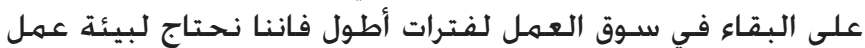

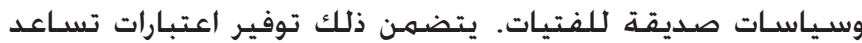

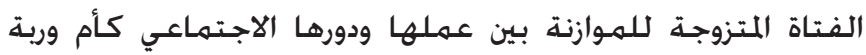

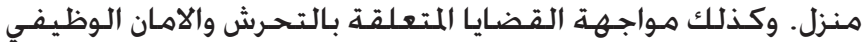

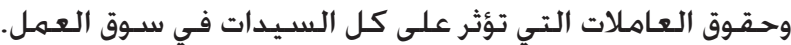

ك"يسين خيـارات العمل المراعيـة لظروف الأسـرة

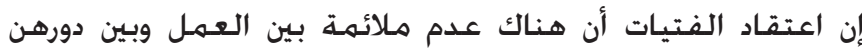

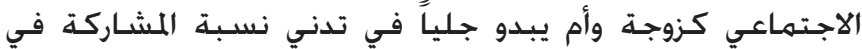

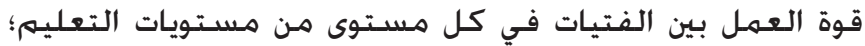

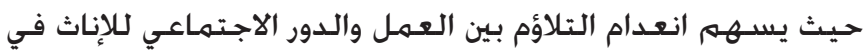

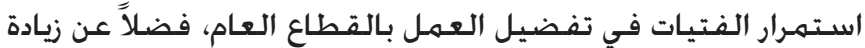

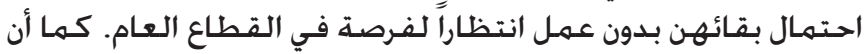

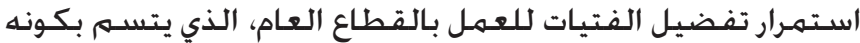

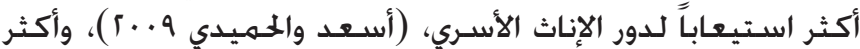

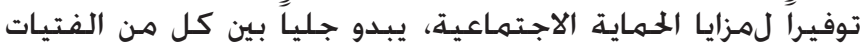

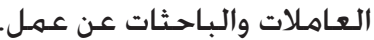

إن خَسـين خيارات العـمل المراعية لظروف الأسـرة في القـاع الخاص

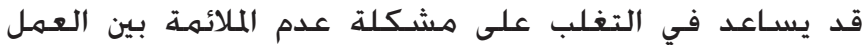

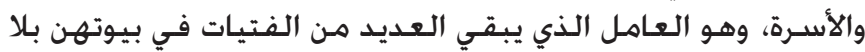

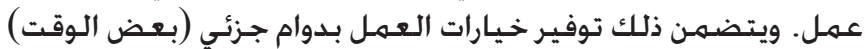

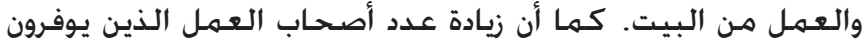

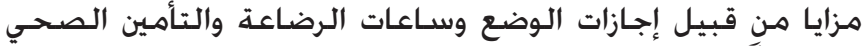

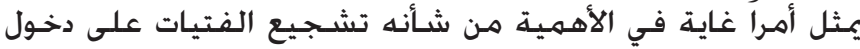

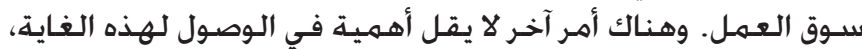

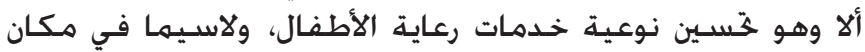

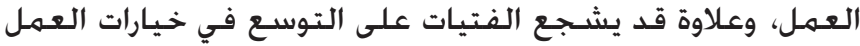

المتاحـة.

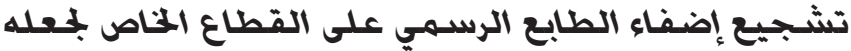
مرحبـا بالسيدات

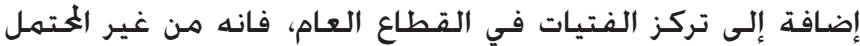

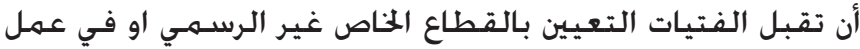

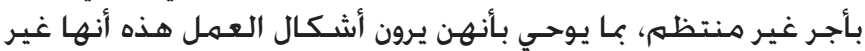

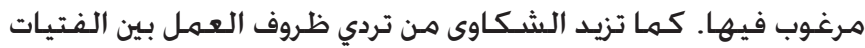

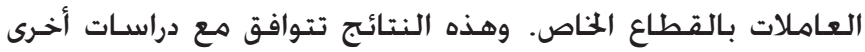

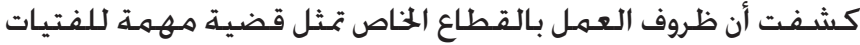

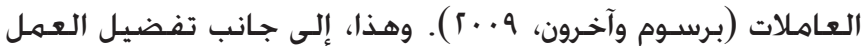

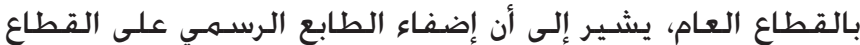


Barsoum, Ghada, Ali Rashed, and Dahlia Hassanien. 2009. When There Is "No Respect" at Work: Job Quality Issues for Women in Egypt's Private Sector. Gender and Work in the MENA Region Working Paper Series: Poverty, Job Quality and Labor Market Dynamics. Cairo: Population Council.

Hausmann, Ricardo, Laura Tyson and Saadia Zahidi. 2011. The Global Gender Gap Report 2011. Geneva: World Economic Forum.

Population Council. 2011. Survey of Young People in Egypt Final Report. Cairo: Population Council.

United Nations Development Program and the Institute of National Planning (UNDP and INP). 2010. Egypt Human Development Report 2010 Youth in Egypt: Building our Future.

The Population Council confronts critical health and development issues-from stopping the spread of HIV to improving reproductive health and ensuring that young people lead full and productive lives. Through biomedical, social science, and public health research in 50 countries, we work with our partners to deliver solutions that lead to more effective policies, programs, and technologies that improve lives around the world. Established in 1952 and headquartered in .New York, the Council is a nongovernmental, nonprofit organization governed by an international board of trustees 